\title{
Double blind study of intrathecal beta-interferon in multiple sclerosis: clinical and laboratory results
}

\author{
C Milanese, A Salmaggi, L La Mantia, A Campi, M Eoli, M Savoiardo, G Bianchi, \\ A Nespolo
}

\begin{abstract}
Sixteen patients with clinically definite MS admitted to a double blind randomised controlled trial of intrathecal natural beta-IFN were followed for a mean of 22 months including the six month treatment period. Clinical response, evaluated in terms of relapse frequency and of progression rate, showed an increase in relapse rate in treated patients during the six month treatment period and, overall, no benefit in treated versus placebo patients. Serial evaluations were made of cerebrospinal fluid (CSF) cells, IgG, myelin basic protein and CSF and blood $T$-cell subsets. $A$ rise in CSF IgG Index, MBP and $\mathrm{DR}+$ cells in IFN-treated patients suggested an activation of intrathecal immune response in treated patients.
\end{abstract}

Interferons (IFNs) have been proposed as a treatment for multiple sclerosis (MS) because of the evidence that a viral infection, an immunoregulatory defect $^{1}$ or both may be implicated in the pathogenesis of the disease. Defective production of type I IFN and reduced natural killer (NK) activity in peripheral blood have been reported in MS patients ${ }^{2-4}$ and it is known that natural beta IFN is a booster of NK activity. ${ }^{5}$

Intrathecally administered beta-IFN has been reported to reduce exacerbation rate in relapsing-remitting MS patients, ${ }^{6}$ while no effect was observed when beta-IFN was given intravenously. ${ }^{7}$ Results with systemic alpha IFN are still controversial, ${ }^{89}$ while gamma IFN has been proven to activate the disease. ${ }^{10}$

To assess the efficacy of intrathecal natural beta IFN in reducing relapse rate or progression of the disease, we carried out a double blind study on MS patients with relapsing and progressive course. Biological activity was assessed by monitoring the following parameters: CSF IgG, cells, IgG-Index, myelin basic protein (MBP), CSF and peripheral blood (PB) T-lymphocyte subsets.

\section{Patients and methods}

Clinically definite $M S,{ }^{11}$ both relapsingremitting (RR) and progressive (P) patients were included, according to the course of the disease in the year preceding the entry. Selection criteria included: active disease (one or more exacerbations per year for RR patients or an increase of one or more points of disability score (DS) in the last two years for $P$ patients), no corticosteroids or immunosuppressive treatment in the previous three and six months respectively, DS at entry less than seven. ${ }^{12}$ Before entry, all patients were regularly followed in our outpatient clinic, most since the onset of the disease or for at least two years. Informed consent was obtained from all patients. The study was approved by the Regional Scientific Committee.

One million units of beta IFN were injected by lumbar puncture twice in the first week, then weekly for the next three weeks and monthly for five months.

At each lumbar puncture CSF was obtained for analysis. Control patients had a sham lumbar puncture (that is, subcutaneous injection of $2 \mathrm{ml}$ of distilled water) according to the same schedule; a true lumbar puncture was performed at the beginning and the end of treatment for CSF analysis. To preserve the blind nature of the trial, both recipient and control patients took indomethacin im $50 \mathrm{mg}$ every six hours on the day of each treatment to reduce the side effects of IFN.

Natural beta IFN (Cytotech-Martigny $\mathrm{CH}$ ) was produced by stimulation of human foreskin fibroblasts (FS 4) by Poly-I, Poly-C and subsequent purification by silicic acid and zinc-chelate chromatography. MRI examination was performed at the beginning and the end of treatment, by a resistive magnet operating at 0.15 Tesla, using the Spin-Echo technique (TR 1000, TE 50, 100, 150 and $200 \mathrm{~ms}$ ); at each examination at least six sections, $10 \mathrm{~mm}$ thick, in the transverse, sagittal and coronal planes, were obtained, according to a standardised protocol to obtain exactly comparable sections. MRI was considered better or worse when a reduction or an increase in size and/or disappearance of old lesions or the appearance of new lesions occurred.

Complete CSF analysis, including MBP quantitation (evaluated by RIA, Diagnostic System Laboratories, Webster, Texas, USA) was performed in treated and control patients at each true lumbar puncture. Peripheral blood and CSF T-lymphocyte subsets (OKT3, T4, T8, NK and DR, antisera Ortho, Raritan, NJ, USA) were concomitantly assessed, as reported elsewhere. ${ }^{13}$ IFN levels were assessed by biological titration (inhibition of infection of human foreskin cells by Indian-type vesicular stomatitis virus).

Statistical analysis was performed by the Mann-Whitney U two tailed test, comparing data in treated and control patients as well as pre- and treatment data in each subgroup. 
Neurological examination with scoring for disability ${ }^{12}$ was performed by a physician blind to the trial on the day preceding each lumbar puncture and every three months after the treatment cycle. An additional neurological assessment was performed for new episodes. An episode is defined as the occurrence of new symptoms and signs, lasting for more than 24 hours. Progression rate was calculated by dividing DS or changes in DS by the relevant period of time in months (overall pre-trial disease duration, or two years preceding the trial, or six months treatment period, or two years follow up).

\section{A) Clinical results}

Sixteen patients were included in the study. Eight (three males and five females) were randomly assigned to IFN, and eight (two males and six females) to the control group. Their clinical data before entry are shown in table 1. Except for the progression rate, which is slightly higher in the control group, no significant differences existed between the two treatment groups.

In February 1989, patients had been followed for 10-24 months: mean follow up was 22.7 months for treated patients and 21 months for controls. Compared with the two year pretrial value, mean relapse frequency (number of episodes/patient/year) during treatment was significantly increased in recipients (from 1.87 to $5, \mathrm{p}=0.01$ ), while it was unchanged in controls. During the six months of treatment, the annual relapse frequency was higher in IFN-treated than in placebo-treated patients $(5$ versus $1.5, p=0.005$ ) (fig 1 ). At the end of the follow up, episode frequency was still higher in recipients than in controls $(2 \cdot 88$ versus $1 \cdot 77$ ) year) but this difference did not reach statistical significance ( $p=0 \cdot 117$ ) (fig 1$)$. During the entire follow up, all patients, both RR and $P$, displayed episodes (frequency range from $0 \cdot 6-$ $5 \cdot 5$ (treated), $0 \cdot 5-5 \cdot 2$ (control)) during the treatment; episodes occurred in all treated patients (from two to 10 per year), and in four of the eight controls (from two to four per year). Two RR patients (one IFN and one placebo) started to progress during the study. At the end of the follow up, deterioration of neurological conditions occurred both in treated (mean DS 5.7) and in controls (mean DS 5.4) $(\mathrm{p}=0 \cdot 48)$. During the period of treatment, increase in DS was more marked in treated patients (from 4.12 to 5.2 ) than in controls (from $4 \cdot 62$ to $4 \cdot 7$ ), but difference was not statistically significant $(p=0.68$ ) (fig 2a). However, more patients in the treated $(7 / 8)$

Table 1 Pretrial clinical data (16 points)

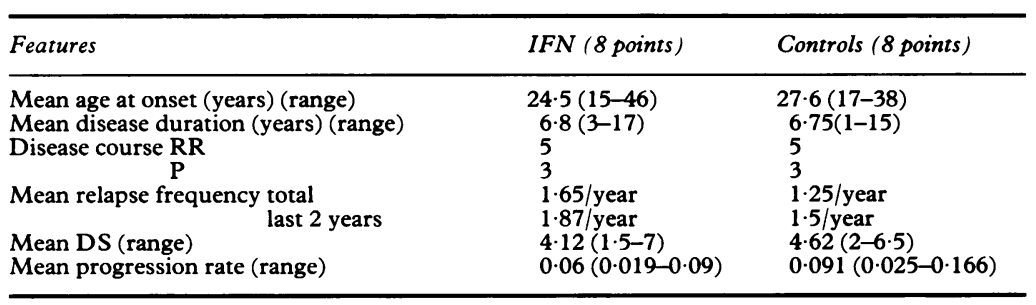

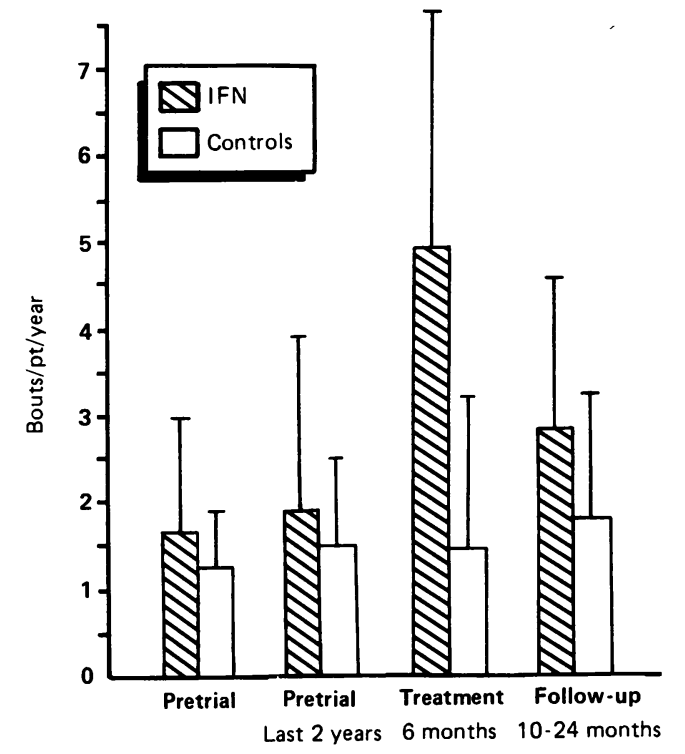

Figure 1 Relapse frequencies (mean and $S D$ ) in treated and control patients before and during treatment and follow up.

than in the control group (5/8) had deteriorated at the end of follow up (fig $2 b$ ).

All six progressive patients (both the three placebo and the three IFN-treated) deteriorated, while of the $10 \mathrm{RR}$ patients, six
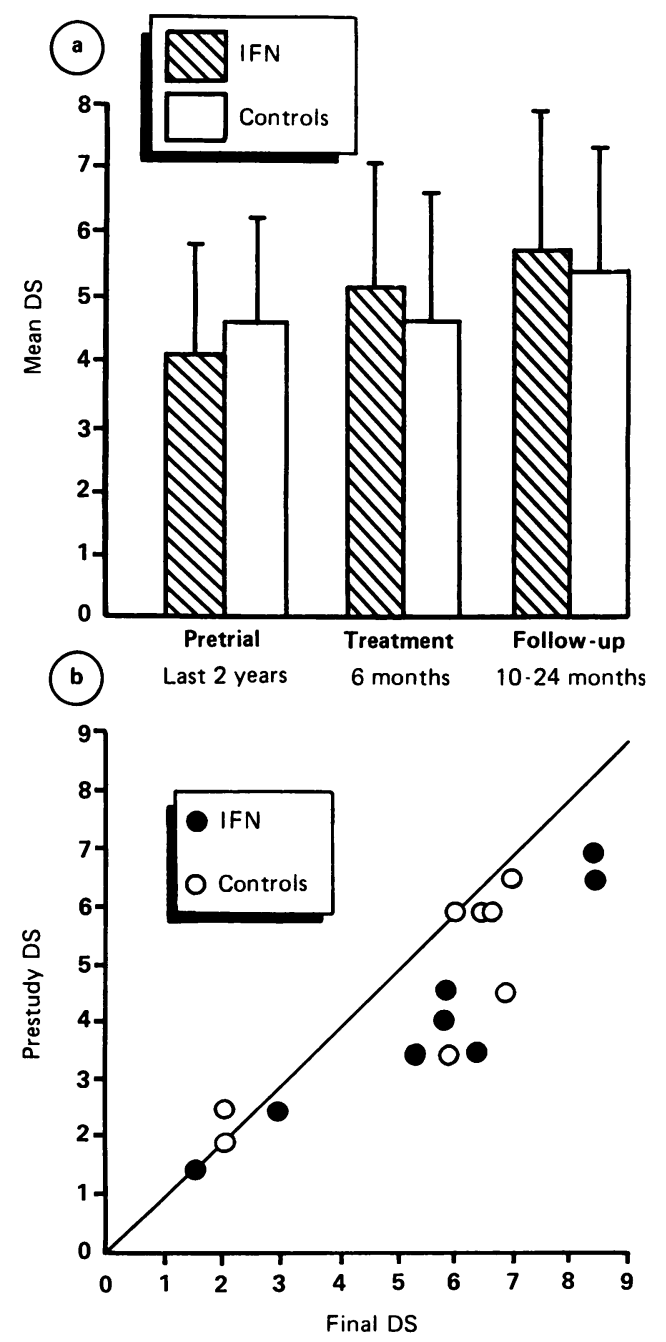

Figure 2 (a) Disability Score (mean and $S D$ ) in treated and control patients before and during treatment and follow up; (b) Comparison of pre-study and final Disability Score in each patient. 
Figure 3 Progression rate (mean and $S D$ ) in treated and control patients before and during treatment and follow up.

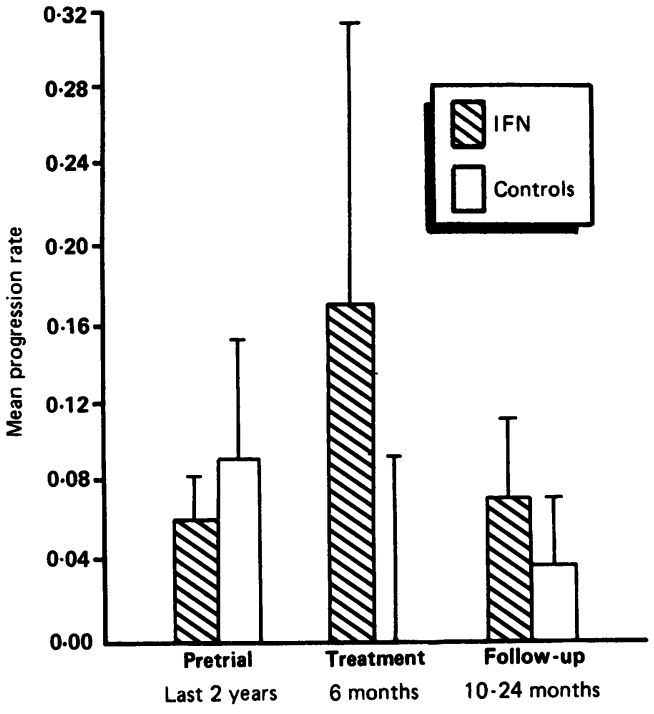

deteriorated (4/5 IFN- and $2 / 5$ placebo). Progression rate increased in recipients during treatment (from 0.06 to 0.17 ), and returned to the pre-study value at the end of follow up $(0.07)$. Compared to the pre-study value the differences were not significant $(p=0.24$ at six months and $p=0.32$ at the end of follow up). However, PR was significantly higher in recipients $(0.17)$ than in controls (0) during treatment, even if care was taken not to assess DS during the episodes (fig 3 ). The difference was significant $(p=0.005)$, due both to deterioration in treated patients and to improvement in controls.

Before treatment, MRI was positive for multiple areas of demyelination in all patients. At the end of the treatment, MRI findings were worse in three and unchanged in five of the recipients, while in the control group they had deteriorated in two, unchanged in five and improved in one. Variation of MRI aspects did not correlate with the clinical evolution both in treated and in control patients.

Side effects were more frequent and more noticeable in treated than in control patients (table 2). In treated patients, fever (less than $38.5^{\circ} \mathrm{C}$ ) subsided within the 48 hours after injection; in some patients two peaks (at six and 30 hours) and in others a single peak (at eight or 24 hours) were observed. Episodes occurring during the six month treatment period were not related to hyperpyrexia. Myalgia, asthenia and nausea were also more frequently reported by treated patients than by controls, and were experienced on more than one occasion. Headache was also an equally frequent complaint in treated and control patients. Despite the higher frequency of side effects in recipients, blindness of the study was not hampered: nine patients thought that they had

Table 2 Side effects

\begin{tabular}{lll}
\hline & IFN (8 points) & Controls (8 points) \\
\hline Fever & 6 & 3 \\
Headache & 6 & 4 \\
Myalgia & 4 & 2 \\
Fatigue & 5 & 1 \\
Nausea and vomiting & 6 & 3 \\
Skin rash & 0 & 2 \\
\hline
\end{tabular}

been treated (five IFN and four controls), five patients thought they had not (two IFN and three controls) and two patients had no opinion (one IFN and one control).

\section{B) Laboratory results}

All treated patients displayed a peak in CSF cells (from 110 to $550 / \mathrm{mm}^{3}$, mostly lymphocytes), within the second week of treatment. The values decreased during the second month and remained low. CSF proteins displayed a slight increase in treated patients, reaching their peak at different times in the various patients. In all treated patients IgG Index increased transiently during the first month, from a mean value of 2.7 to 3.9 in those with an abnormal baseline Index, and from 0.7 to $1 \cdot 1$ in those with a normal baseline value.

A rise of the mean value of CSF MBP was observed during the first month of treatment (from $1.98 \mathrm{ng} / \mathrm{ml}$ to $4.09 \mathrm{ng} / \mathrm{ml}$ ), after which mean values decreased steadily until the end of the six month cycle of treatment (mean value $2.37 \mathrm{ng} / \mathrm{ml}$ ). CSF MBP values changed during treatment following two main patterns: 1) in four patients, all with relapsing-remitting course, MBP levels rose and had their peak within the first month of treatment, falling to nearly baseline levels at the end of the treatment; 2) in four patients, three with a progressive and one with a relapsing-remitting course turning to progressive during the study, MBP levels showed little change during the first month, but rose thereafter in three.

Mean values of blood $T$-cell subsets were similar in treated and control patients, before and during treatment, except for a transient decrease of $\mathrm{T} 4 / \mathrm{T} 8$ ratio seen in treated patients, three days after the first IFN administration. As far as CSF T4/T8 ratio is concerned, progressive patients displayed wideamplitude fluctuations more often than RR patients, regardless of treatment. CSF NK cells were low (less than 5\%) before and after treatment both in treated and control patients. In all treated patients CSF NK cells were lower than the PB; however, transient rises occurred in six patients, in four after the third and in two within the first month of treatment. In individual patients CSF NK values did not parallel $P B$ changes.

CSF DR + cells were similar in treated and control patients before $(10 \cdot 1 \%$ and $15.8 \%)$ and after $(14.2 \%$ and $11.37 \%)$ treatment. In individual patients marked fluctuations of CSF $\mathrm{DR}+$ cells were observed during treatment. When mean values of CSF DR + cells in treated patients were expressed as a percentage of baseline values, a marked rise was detected after the fourth lumbar puncture (fig 4). In individual patients no clear correlation was found between $T$-cell subset fluctuations and clinical exacerbations.

Beta-IFN concentrations were analysed in CSF and serum in two treated and three control patients: concentrations in CSF reached a peak at the second lumbar puncture in treated patients, but fell quickly to be undetectable at the sixth and eighth lumbar puncture. IFN was 
Figure 4 Mean $C S F$ $D R+$ cells variation, expressed as percentage of baseline values, in IFNtreated patients.

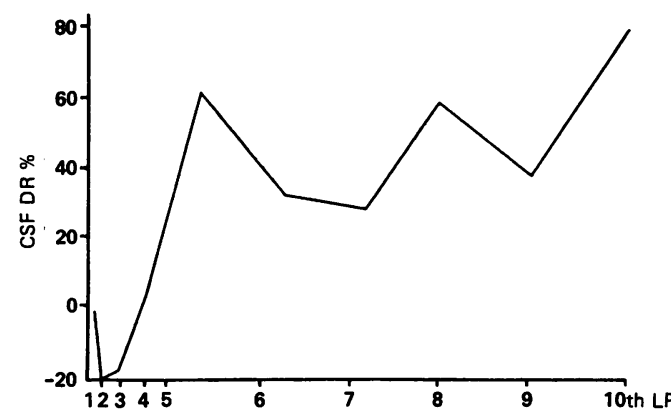

detected in the serum of one placebo patient from the third lumbar puncture to the end of treatment and in two other placebo patients on just one sample.

\section{Discussion}

The clinical results of our study conflict with the favourable response to intrathecal betaIFN reported by Jacobs. ${ }^{6}$ Both episode frequency and progression of disability increased in IFN-treated patients during the trial. Different results cannot be ascribed to treatment: we used the same type of IFN, the same timing, dose and route of administration as Jacobs. Our preparation was purified by silicic acid and zinc-chelate chromatography, yielding an activity of $2 \cdot 10^{7} \mathrm{IRU}$ (International Reference Units) of beta-IFN/mg of protein. It is possible that minor differences in the purification protocol can account for differences in clinical results.

One possible explanation might reside in patients' selection: all patients in Jacob's study had a relapsing-remitting course, while six of our patients had a progressive course. In a previous study by $\mathrm{Knobler}^{8}$ with im alphaIFN, a clear reduction in relapse frequency was observed only in purely exacerbating-remitting patients and not in exacerbating-progressive ones. However, we observed an increase in relapse frequency in $R R$ patients and appearance of relapses in chronic progressive ones. The increase we observed in relapse rate occurred mostly during the six month treatment period, agrees with the report by Jacobs for the first two months. Since our mean follow up is 22 months, it is possible that a further reduction in relapse rate could be seen in a follow up of two years as in the study by Jacobs.

IFNs induce expression of Class I and Class II antigens and of several other surface markers on macrophages, enhancing immune activity. ${ }^{14}$ It is well known that the HLA antigens' association with MS in Italian patients are different from those reported in other populations. ${ }^{15}$ Different HLA haplotypes may imply differences in response to exogenous IFNs. Surprisingly, our results closely resemble those obtained by Panitch et al with systemic iv gamma-IFN. ${ }^{10}$ Our immunological data confirm that intrathecal administration of natural beta-IFN evokes an acute meningitic reaction with lymphocytic pleocytosis (mostly during the first week) and a rise in CSF total protein. Furthermore, the sharp rise in CSF MBP in remitting patients, the transient rises in IgG Index, in CSF NK + cells and the late increase in CSF DR + cells in treated patients, point to an IFN-induced intrathecal immune activation.

Recent evidence ${ }^{16}$ suggests that beta-IFN in vitro may exert an antagonistic dose-dependent effect on gamma-IFN induced expression of Ia antigen in murine macrophages. This effect is seen only when beta-IFN is present either before or together with gamma-IFN, while no effect is seen if gamma-IFN has been added before.

These interrelationships might explain fluctuations in biological activity of beta-IFN depending upon the presence of in vivo gamma-IFN, the production of which is still a matter of debate in MS. ${ }^{21718}$ Due to the conflicting results of trials with IFNs, and to the incomplete knowledge of their mechanisms of action in MS, the therapeutic use of IFNs in MS remains questionable. Further studies are necessary to evaluate the therapeutic efficacy of IFNs in MS. Careful immunological monitoring will help to improve the knowledge of the mechanisms of action and to select the likely responders to IFN therapy.

1 Waksman B. Mechanism in multiple sclerosis. Nature 1985;318:104-5.

2 Neighbour PA, Miller AE, Bloom BR. Interferon response of leukocytes in multiple sclerosis. Neurology (NY) 1981;31:561-6.

3 Neighbour PA. Studies of interferon production and natural killing by lymphocytes from multiple sclerosis patients. In: Scheinbergh, Raine CS, eds. Multiple sclerosis: experimental and clinical aspects. Ann NY Acad Sci experimental and

4 Merrill JE, Gerrer RH, Myers LW, Ellison GW. Regulation of natural killer cell cytotoxicity by prostaglandin $E$ in the peripheral blood and cercbrospinal fluid of patients with multiple sclerosis and other neurological diseases. $J$ Neuroimmunol 1983;4:223-37.

5 Brunda MJ, Rosenbaum D. Modulation of murine natural killer cell activity in vitro and in vivo by recombinan human interferons. Cancer Res 1984;44:597-601.

6 Jacobs L, Salazar AM, Herndon R, et al. Multicentre double blind study of effect of intrathecally administered natural human fibroblast interferon on exacerbations of multiple sclerosis. Lancet 1986;ii:1411-3.

7 Huber M, Bamborschke S, Assheuer J, Heiss WD. Intravenous natural beta interferon treatment of chronic exacerbating-remitting multiple sclerosis: clinical response and CSF/MRI findings. $J$ Neurol 1988;235:171-3.

8 Knobler RL, Panitch HS, Branheny SL, et al. Systemic alpha- interferon therapy of multiple sclerosis. Neurology 1984;34:1273-9.

9 Camenga DL, Johnson KP, Alter M, et al. Systemic recombinant alfa-2 interferon therapy in relapsing multiple sclerosis. Arch Neurol 1986;43:1239-46.

10 Panitch HS, Hirsch RL, Schindler J, Johnson KP. Treatment of multiple sclerosis with gamma interferon: exacerbations associated with activation of the immune system. Neurology 1987;37:1097-102.

11 McDonald WI, Halliday AM. Diagnosis and classification of multiple sclerosis. Br Med Bull 1977;33:4-9.

12 Kurtzke JF. Rating neurological impairment in multiple sclerosis: an expanded disability status scale (EDSS). Nerosis: an expanded disa

13 Salmaggi A, La Mantia L, Milanese C, et al. CSF T-cells subsets in multiple sclerosis: relationship to CSF myelin basic protein and clinical activity. $J$ Neurol 1989;236: 336-9

14 Wallach D, Fellous $M$, Revel $M$. Preferential effect of interferon on synthesis of HLA antigens and their mRNAs in human cells. Nature 1982;299:833.

15 McDonald WI. Multiple sclerosis: epidemiology and HLA associations. In: Scheinberg L, Raine CS, eds. Multiple sclerosis: experimental and clinical aspects. New York: Ann NY Acad Sci, 1984:436:109-17.

16 Ling PD, Warren MK, Vogel SN. Antagonistic effect of interferon beta on the interferon-gamma induced expression of Ia antigen in murine macrophages. J Immunol 1985;135:1857-63.

17 Vervliet $G$, Claeys $H$, Van Haver $H$, et al. Interferon production and natural killer (NK) activity in leukocyte
cultures from multiple sclerosis patients. $J$ Neurol $S c i$ cultures from mult

18 Hirsch RL, Panitch HS, Johnson KP. Lymphocytes from multiple sclerosis patients produce elevated levels of gamma interferon in vitro. J Clin Immunol 1985;5(6): 